\title{
PROBLEMÁTICA JURÍDICA DE LA PRODUCCIÓN GANADERA SUSTENTABLE COMO FORMA DE MITIGAR EL CAMBIO CLIMÁTICO
}

\author{
LEGAL PROBLEMS IN SUSTAINABLE LIVESTOCK PRODUCTION AS A WAY OF \\ MITIGATING CLIMATE CHANGE
}

Martín Miguel Chalup

(Universidad Nacional del Nordeste / Argentina)

martin.m.chalup@gmail.com

Diego Eduardo Bissaro Fava

(Universidad Nacional del Nordeste / Argentina)

diemar.ciudad@gmail.com

Recibido: $11 / 09 / 2018$

Aprobado: 30/10/2018

\section{RESUMEN}

Las actividades agropecuarias, a nivel global tienen un impacto significativo prácticamente en todas las esferas del ambiente y recursos naturales. La ganadería es uno de los sectores económicos con repercusiones más graves en el ambiente, en todos los niveles, desde el local a lo mundial. En Corrientes ésta representa una de las principales actividades. En el presente trabajo abordamos la problemática de la producción ganadera en la provincia de Corrientes y reflexionamos acerca de cómo los instrumentos jurídicos que regulan ésta actividad colaboran con mitigar el cambio climático y promover una actividad ganadera sustentable. Para el trabajo utilizamos una metodología cualitativa, exploratoria, nos basamos en la observación del fenómeno, acompañado de descripciones, mediciones de organismos públicos y análisis comparativo. Las técnicas que se utilizaron fueron la revisión y exploración histórica y contextual de las normas e instrumentos jurídicos identificados. Primero abordamos la problemática desde el ámbito nacional, para luego ceñirnos en la regulación particular provincial. Por ultimo pudimos identificar mecanismos o instrumentos normativos alternativos. Esta revisión nos permitió detectar que en el ámbito de Corrientes existen normativas específicas referidas a áreas protegidas y a bosque nativos que establecen límites a la ganadería, con el fin de compatibilizar con la protección al ambiente. Sin embargo estas regulaciones no dan respuesta definitiva y solo dan soluciones parciales a las fuertes presiones que la legislación internacional demanda. Por otro lado los programas de promoción de la ganadería integrada y la certificación privada revalorizan la inclusión del recurso árbol en sistemas agro-ecológicos con énfasis en la captura de carbono, pero no quedan libres de cuestionamientos.

Palabras Claves: Actividad - Ambiente - Desarrollo Sostenible

\section{ABSCTRAC}

Agricultural activities, at the global level have a significant impact practically in all spheres of the environment and natural resources. Livestock is one of the economic sectors with more serious repercussions on the environment, at all levels, from local to global. In Corrientes, livestock represents one of the main activities. In this paper we address the 
problem of livestock production in the province of Corrientes and reflect about how the legal instruments that regulate this activity collaborate with mitigating climate change and promoting a sustainable livestock production. We use a qualitative, exploratory methodology, based on the observation of the phenomenon, accompanied by descriptions, measurements of public offices and comparative analysis. The techniques used were review and historical and contextual exploration of identified legal instruments and norms. First we address the issues from the national level, and then to focus on the particular provincial regulation. Finally, we were able to identify mechanisms or alternative policy instruments. This review allowed us to detect that there are specific rules in the field of streams referred to protected areas and forest natives to establish limits on livestock, in order to make compatible with the protection of the environment. However, these regulations do not give a definitive answer, not comprehensively address the problem of climate change and not respond to the strong pressures that international law demands. On the other hand the promotion of integrated livestock and private certification programs revalued the benefits that has the inclusion of trees in agro-ecological systems, mainly on carbon sequestration, but are not free of questioning.

Keywords: Activity - Environment - Sustainable Development

\section{Introducción}

La creciente demanda de alimentos y el aumento de tierras productivas nos obliga a pensar en un vínculo estrecho, entre la producción agropecuaria y la emisión de gases de efectos invernadero (GEI). Entonces, ¿Cómo se relaciona el cambio climático con la producción ganadera? Desde principio de tiempos el hombre transformo continuamente su entorno, logrando avances, pero también, altero ciclos propios de la naturaleza.

La producción agropecuaria es uno de los agentes determinantes del cambio climático que en los últimos años aumentó las proporciones de tierras cultivables en los países en vía de desarrollo y disminuyó en los países más desarrollados. Sector que responde a una demanda cada vez mayor y repercute directamente en los niveles de emisiones, que en los últimos años aumentó el 17\% a nivel mundial. En Argentina en el 2002 por ejemplo el 44\% de las emisiones provinieron del sector agropecuario y del cual el 60\% corresponden al ganado bovino (Santini \& Senetiner, 2011).

Las actividades tanto agrarias como pecuarias en general tienen un gran impacto significativo, prácticamente en todas las esferas del ambiente, incluyendo en el cambio climático. La ganadería es uno de los sectores económicos con repercusiones más graves en el ambiente, en todos los niveles, desde el local a lo mundial. En Argentina, esta actividad contribuye directamente a la emisión de GEI aportando el 35\% del total del País. Si bien no existe actualmente una exactitud para saber cuánto GEI emite un ganado en forma diaria, es posible obtener un aproximado a los valores de niveles de contaminación. Por ejemplo un bovino de $500 \mathrm{Kg}$ produce unos 1000 litros de gases diarios, de los cuales el $20 \%$ es gas metano. Este gas permanece en el ambiente (atmosfera) de 9 a 15 años y el poder de retención de calor ronda unas 21 veces superior al carbono en un siglo (Santini \& Senetiner, 2011; Steinfeld et al., 2009).

La práctica de la ganadería cuando es realizada de forma que sobrepasa la capacidad de recuperación del suelo y vegetación, produce consecuencias negativas al ambiente. En esta actividad, denominada sobrepastoreo, el pisoteo produce una modificación de los horizontes del suelo, generando y/o contribuyendo a fenómenos de erosión hídrica o eólica, que en algunos casos, lleva a procesos de desertificación y en ambientes vulnerables esta práctica degrada los suelos y la biodiversidad, disminuyendo el stock provocando la insostenibilidad de la práctica. (M. de A. y D. S.-R. Argentina, 2016). 
Si bien la evolución de la cantidad de cabezas de ganado bovino en Argentina tuvo un incremento hasta los años 60, y desde entonces permaneció estable (alrededor de 50 millones de cabezas), son de destacar los cambios respecto a la distribución geográfica. En las últimas décadas se registró un incremento del desplazamiento de la producción hacia las Provincias del norte del país, históricamente concentrada en la región Pampeana. Este desplazamiento se debe a la técnica de producción en feedlots aplicado al ganado bovino, así como la presión ejercida por otros usos del suelo, como la actividad agrícola por ejemplo la sojera que necesita el 100\% de los suelos y no podría ser compatible con el pastoreo (M. de A. y D. S.-R. Argentina, 2016).

En la región NEA de Argentina, encontramos áreas de más de 30 millones de hectáreas con gran diversidad de suelos y vegetación, pero con un componente común, la presencia del árbol. Dentro de la región mencionada la selva Misionera ocupa gran parte junto al bosque del parque chaqueño y también encontramos los suelos arenosos de la provincia de Corrientes y Entre Ríos (Mahecha, 2002; Joaquin \& Pérego, 2001)

En Corrientes la ganadería es una de las principales actividades económicas donde aproximadamente, pastan 4.165.000 bovinos y 2.292.000 cabezas de ganado ovino, esto tiene relevancia en la ganadería porque su stock representa el 9,9\% y $36 \%$ de las existencias bovinas a nivel nacional y del NEA. La actividad se desarrolla en la región conjuntamente con el monte natural y más recientemente como actividad complementaria en los montes implantados. Esta combinación se la define como Sistema Silvopastoril el cual es un "sistema sostenible de manejo de suelos, incrementándose el potencial productivo del mismo, ya que combina la producción de forrajeras, naturales o implantadas, árboles y animales sobre un mismo terreno" (Mahecha, 2002). Estudios realizados en Corrientes, comprobaron que 1 ha de producción conjunta de ganado y pasturas compensa la emisión de metano de cinco cabezas de ganado (Agritotal, 2017).

En el presente trabajo proponemos abordar la problemática de la producción ganadera en la provincia de Corrientes y reflexionamos acerca de ¿Cómo los instrumentos jurídicos que regulan ésta actividad colaboran con mitigar el Cambio Climático y promover una actividad ganadera sustentable? Para ello utilizamos una metodología cualitativa, exploratoria, nos basamos en la observación del fenómeno, acompañado de descripciones, mediciones de organismos públicos y análisis comparativo. Las técnicas que se utilizarán serán la revisión y exploración histórica y contextual de las normas e instrumentos jurídicos identificados.

Realizamos un abordaje de la situación actual de las normas y estándares que pretenden regular la actividad en las diferentes áreas y regiones, desde un plano nacional partiendo por nuestro ordenamiento fundamental, refiriendo a los convenios y tratados para desembarcar en la legislación local. Después de haber explorado y analizado críticamente las distintas fuentes de la actividad y las normas jurídicas, nos centramos en describir los acuerdos públicos y privados y modalidades de la actividad.

\section{Panorama jurídico Nacional}

El ambiente como bien jurídico protegido se estructura positivamente en un conjunto de normas, que simbólicamente, se consagran en múltiples instrumentos legales de diferente naturaleza que complejizan su materialidad y concreción. La norma central es el art. 41 de la Constitución Nacional de 1994 que consagra el derecho-deber a un ambiente sano, conjuntamente con los principios de preservación y de reparación, y desarrollo sustentable al establecer que deben satisfacer "las necesidades presentes sin comprometer las de las generaciones futuras". Coincidente con el concepto del informe de Brundtland, "Nuestro Futuro Común" (Brundtland, 1987). Teniendo como fuente los principales resultados de las conferencias y convenciones internacionales hasta 1994, generándose así un nuevo paradigma en el sistema productivo al que debe ajustarse la actividad económica. También el art $41 \mathrm{CN}$ impone a las autoridades como marco en la actividad productiva, proveer la "utilización 
racional de los recursos naturales", la "preservación del patrimonio natural" y "diversidad biológica" (Casella, 1997).

Por otra parte, el párrafo tercero del art. 41, dispone la "competencia nacional de dictar las normas que contengan los presupuestos mínimos de protección ambiental sin que aquellas alteren las jurisdicciones locales". Correlativamente, encomienda a las provincias dictar las normas para complementarlas, derivando en una delegación a favor de la Nación en lo que hace a la determinación de presupuestos mínimos (Sabsay \& Di Paola, 2002).

Pero el constituyente considero conveniente incorporar un límite a dicha competencia en el art. 124 de la C.N., al expresar que "corresponde a las provincias el dominio originario de los recursos naturales existentes en su territorio". Así el ejercicio de las facultades de la nación en dictar dichas normas no puede importar un vaciamiento del dominio que tienen las provincias sobre los recursos naturales en su territorio (Sabsay \& Di Paola, 2002). Esta distribución de competencia recibió el nombre de federalismo de concertación (Sabsay, 1997) o federalismo ambiental; para referirse al cambio en la forma de distribuir el poder vertical del Estado, en la concurrencia competencial entre Nación y Provincias (Esain, 2008).

A partir de la reforma constitucional de 1994 se abre camino a la internacionalización del derecho internacional (Agustín Alberto Gordillo, 2007) y nuestra Constitución reconoce a los tratados como normas vigentes en nuestro derecho en el art. $31^{1}$; Pero los ubica por encima de las leyes en el art. 72 inc. 22 generando que los tratados en materia de protección ambiental y recursos naturales adquieran una jerarquía como normas internas y superior a las leyes, siempre que estos tratados hayan sido ratificados por ley del Congreso. Esto da lugar a la ampliación de principios, reglas, restricciones e instrumentos en materia de derecho ambiental conformando un complejo entramado de normas que comprenden el bien jurídicamente protegido del ambiente.

Específicamente en materia de cambio climático en el orden internacional la temática se cristaliza con la Convención Marco de las Naciones Unidas sobre Cambio Climático (CMNUCC). Convenio que se abrió a firmas en la Conferencia de Río de Janeiro de 1992 a la que Argentina adhiere con la sanción de la ley 24295 (Congreso de la Nación Argentina, 1994).

El CMNUCC estaba basado en la equidad, traducida por el principio de responsabilidades comunes pero diferenciadas, siendo su objetivo último estabilizar las concentraciones de GEI en la atmosfera a un nivel que impida injerencia en un plazo razonable que permita a los ecosistemas recuperar su capacidad de carga y/o adaptarse naturalmente al cambio climático (Cafferatta, 2012), así brindar "un marco de cooperación de los Estados en orden a facilitar las acciones de mitigación." (Lucas Garín, 2017).

Dicho convenio, en su el art. 4 impone obligaciones a todos los Estados Parte: i) preparar inventarios nacionales de sus emisiones, ii) tomar medidas nacionales y regionales para atenuar dichas emisiones iii) adaptarse a los efectos del cambio climático, y iv) cooperar a estos fines (Cafferatta, 2012).

En 1997 se aprueba el Protocolo de Kioto, como acuerdo vinculado a la CMNUCC, con mayores exigencias elevando los compromisos de limitación y reducción de GEI.; El cual Argentina en 2001 lo aprobó como ley interna No 25438 (Congreso de la Nación Argentina, 2001)

El protocolo estableció una serie de compromisos vinculantes para las principales economías mundiales que lo hayan suscrito. Sin embargo, el modo para cumplirlo es flexible. De esta manera posibilita tres mecanismos para ayudar a las partes y promover el desarrollo sustentable: i)

\footnotetext{
1 "La Constitución de 1853/60 establecía un orden de prelación dentro del ámbito de su competencia territorial y en consonancia a los tiempos de su dictado; esa norma, más de un siglo después, debe ser entendida en consonancia con la jerarquía actualmente vigente de normas" (Gordillo, A., 2007 y N.D)
} 
implementación conjunta ii) régimen de venta de emisiones y iii) mecanismo de desarrollo limpio (MDL) (Restrepo, Tobón, \& Flórez, 2008; Rodríguez, 2007).

Los Estados pueden compensar parcialmente sus emisiones de GEI aumentando los sumideros, a partir de la implantación de bosques que suprimen el carbono de la atmósfera, lo cual es beneficioso para todos. Pueden, también, financiar proyectos en el extranjero que tengan por objeto reducción de GEI. Se establecieron varios mecanismos para el comercio de las emisiones, o mercado del carbono. Así ésta permitido que los países que no utilicen la totalidad de sus unidades de emisión, puedan vender el excedente de capacidad a los que superan sus objetivos y los país que no alcanzaran a cumplir sus compromisos pueden comprar (Restrepo et al., 2008; Steinfeld et al., 2009)

En esta lógica de mercado, se pueden obtener créditos para reducir la totalidad de GEI plantando bosques y/o ampliando la superficie forestal. Así como realizando "proyectos de aplicación conjunta" con otros países para reducir las emisiones. Los créditos obtenidos pueden comercializarse en el mercado de emisiones o reservar para el uso futuro (Steinfeld et al., 2009)

Los MDL permiten a los países desarrollados financiar proyectos con el objeto de reducir o evitar emisiones en los países no obligados y a cambio se otorgan créditos para ser utilizados en el cumplimiento de mecanismos alternativos de emisión y los receptores se benefician de la tecnología suministrada.

Con la intensión de reforzar los esfuerzos antes asumidos y en búsqueda de renovar responsabilidad, se llevó a cabo en 2016 el Acuerdo de Paris. Constituyéndose en un instrumento jurídico formado por una combinación de disposiciones jurídicamente vinculantes como no vinculantes (Victoria, 2017). Un instrumento hard law con un alto contenido de normas soft law (Nava Escudero, 2016). Argentina firmó y ratifico el Acuerdo por Ley 27.270 en 2016 (Congreso de la Nación Argentina, 2017).

Este acuerdo mantiene la visión de responsabilidad diferenciada pero común del CMNUCC; Sin embargo de una manera diferente adopta metas absolutas de reducción de emisiones para los países desarrollados, mientras que las Partes que son países en desarrollo deberían seguir aumentando sus esfuerzos de mitigación (Victoria, 2017). La principal diferencia radica que todos los Estados Parte, se encuentran obligados a presentar sus contribuciones previstas y determinadas a nivel Nacional y deberán realizarla cada cinco años (Garín, 2017). Cada Parte establece cómo será su aporte teniendo presente una "auto-calificación de justicia y ambición” (Bueno Rubial, 2016).

En esta descripción de normas, es importante agregar que a partir de 2002, la Nación ejerció su facultad de dictar normas de presupuestos mínimos como; las leyes $25.675 ; 25.688 ; 25.831 ; 26.331$ y 26639 entre otras (Congreso de la Nación Argentina, 2002, 2003a, 2003b, 2007, 2010). Específicamente la ley 25.675 que normativiza un orden publico ambiental y del desarrollo sustentable reglamentó la implementación y promoción del desarrollo sostenible y los principios e instrumentos de política y gestión ambiental.

\section{Panorama jurídico aplicable en la Provincia}

En nuestra búsqueda para el análisis y estudio de un instrumento o norma jurídica que regule de manera específica e integral la actividad ganadera en la provincia de Corrientes que promueva una actividad productiva sustentable como alternativa al cambio climático, nos hemos encontrado con su negativa o total ausencia. Sin embargo, al observar el sistema jurídico local en su totalidad, pudimos identificar normas locales que de manera dispersa y con diferente naturaleza y prelación, restringen, limitan y califican a la actividad ganadera en el ámbito de la Provincia.

Particularmente estas normas identificadas tienden a asociar la actividad al ámbito espacial donde es desarrollada. Por lo tanto, solamente serán aplicables a ciertos sectores ecosistémicos particulares de la Provincia. Esto genera una incongruencia entre el sistema jurídico normativo y la realidad al 
presuponer que los ecosistemas, los posibles daños y pasivos ambientales, presuponen límites jurídicos y políticos. Provocando una segmentación y truncamiento de la actividad sustentable ganadera en relación al ámbito espacial. Estas normas jurídicas identificadas son las que refieren a la actividad ganadera en zona de reserva y en bosque nativo, siendo esta segunda más amplia o general que la primera, pero a su vez ambas íntimamente relacionadas.

En Corrientes los Esteros del Iberá constituyen una categoría de gran relevancia no solo por su gran importancia ecosistémica, sino también por su escala económica, política y jurídica. Ocupan el centro norte de la provincia sobre el Acuífero Guaraní, y constituyen un importante reservorio en estado natural de agua dulce con ubicación estratégica mundialmente y el humedal más importante de Argentina $^{2}$ y el segundo de Sudamérica por su extensión y sus particularidades biogeográficas (Vallejos, Botana \& Pohl, 2009).

La Constitución de Corrientes reformada en 2007 en su art 66 otorga protección especial a esta zona, declarándola como Patrimonio Estratégico Natural y Cultural del Estado y Bien del Dominio Público de la provincia, "a los fines de su preservación, conservación y defensa: el ecosistema Iberá, sus esteros y su diversidad biológica, y como reservorio de agua dulce..."(Convención Constituyente de la Provincia de Corrientes, 2007).

Normativamente la reversa Iberá fue creada en 1983, por ley 3771 (Gobernador de la Provincia de Corrientes, 1983). Pero es recién a partir de la ley 4736/93 (Senado y la Cámara de Diputados de la Provincia de Corrientes, 1993) que se establece un sistema general de áreas protegidas en la Provincia, por un lado se crea el Parque Iberá, otorgándole una protección absoluta -art. 5-, por la que deberán conservarse en su estado natural, quedando prohibido todo tipo de actividad que pueda modificarlo, prohibiéndose las explotación pecuaria y cualquier otro aprovechamiento de los recursos naturales art. 6-.

Sin embargo respecto de la categoría Reservas Naturales -art. 9- permite realizar ciertas actividades productivas cuando sean compatible con la conservación de los recursos naturales y que permitan el uso racional de los mismos sin afectar las características más relevantes e importantes del área o su equilibrio ecológico. En el decreto $\mathrm{N}^{\circ} 1440 / 09^{3}$ (Gobernador de la Provincia de Corrientes, 2009a), se reglamentan las restricciones a las actividades permitidas a través de estándares generales y particulares, cuando esta actividad por sus características y/o escala superficial, implique un efecto notable dentro del área de la Reserva, deberán observar ciertas limitaciones.

Así por ejemplo, en la ganadería extensiva realizada sobre áreas de pasturas naturales deberán por un lado, dar cumplimiento a las leyes provinciales de "Bosques Nativos" № 5974 (2010) y de "Manejo y Control del Fuego" N 5590 (2004), como también se deberá cumplir una conservación mínima de los suelos con su vegetación natural, integrar la superficie destinada a la producción con corredores biológicos o franjas de amortiguación en las áreas de humedales y bosques nativos para lograr su continuidad espacial. Por ejemplo mantener $70 \mathrm{mts}$ circundantes al límite exterior del estero, bañado o planicie de inundación del valle aluvial del río principal del sistema (Gobernador de la Provincia de Corrientes, 2009a).

La ley $\mathrm{N}^{\circ} 26.331$ de Presupuestos Mínimos de Protección Ambiental de los Bosques Nativos (Congreso de la Nación Argentina, 2007), reglamentada mediante el Decreto 91/09 (PEN, 2009), genero un verdadero instrumento de límite a la propiedad sobre bosques nativos (BN), imponiendo a las Provincias la obligatoriedad de sancionar su ordenamiento territorial de BN (OTBN) existente en su territorio mediante un proceso participativo, de acuerdo a los criterios de sustentabilidad. Corrientes

\footnotetext{
${ }^{2}$ En enero de 2002 por ley 23919 (Congreso de la Nación Argentina, 1991), se incorporaron como sitio Ramsar de la Argentina a un punto central del Macrosistema del Iberá.

${ }^{3}$ Específicamente en lo referente al procedimiento administrativo del Estudio del Impacto Ambiental ha sido modificada por el Decreto 1966/09 (Gobernador de la Provincia de Corrientes, 2009b).
} 
sancionó su OTBN por ley provincial 5.974 (2010), complementando la ley nacional, conformando un régimen de protección nacional-provincial.

El régimen establece tres categorías de conservación en función del valor ambiental de los $\mathrm{BN}$ y sus servicios ambientales. Las cuales a su vez imponen diferentes restricciones en el uso y goce de los BN. Dichas categorías se clasifican, en:

I -rojo- de muy alto valor de conservación, merecen su persistencia como bosques a perpetuidad. No pueden alterarse y solo pueden realizarse actividades que no modifiquen sus atributos, como actividades de recolección, turismo responsable e investigación.

II-amarilla- mediano valor de conservación, no pueden ser sometidos a cambios en el uso del suelo. Se encuentra prohibido el desmonte, pero se permite, un uso de manejo sostenible.

III -verde- de bajo valor de conservación y pueden transformarse parcialmente o en su totalidad. Es decir está permitido el desmonte previa autorización.

En total se zonificaron 770.319 ha de BN, 63.840 ha pertenecientes a la cat. I roja (8\%); 292.251 ha cat. II amarilla (38\%) y 414.228 ha a la cat. III verde (54\%) (Área de Ordenamiento Territorial, Área de Vinculación y Control, \& Ministerio de Ambiente y Desarrollo Sustentable de la Nación, 2015). Es decir que en gran parte del territorio de $\mathrm{BN}$-más del 50\%- es posible realizar ganadería.

La actividad ganadera en términos generales es comprendida dentro del concepto de desmonte. Por lo que solo estaría permitida en los BN cat. III. Pero en esta situación, cabe preguntarse si es posible realizar actividad ganadera en la cat. II, cuando dicha actividad sea compatible con un manejo sustentable (Carranza, 2016; Laclau, 2012; Preliasco \& Mañarro, 2016). Entendido por tal a la organización, administración y uso de los $\mathrm{BN}$ "que permita mantener su biodiversidad, productividad, vitalidad, potencialidad y capacidad de regeneración, para atender, ahora y en el futuro, las funciones" y servicios ambientales importantes a nivel local y nacional, sin producir daños a otros ecosistemas (Poder Legislativo de Corrientes, 2010).

\section{Medidas alternativas de producción de ganadera}

Ante los posibles problemas ambientales generados por las actividades agropecuarias y en particular por ganadería se fueron promoviendo, desde el ámbito técnico-practico sistemas alternativos de producción, con la finalidad de mejorar la sustentabilidad (Silberman, Albanesi, \& Grasso, 2016). Principalmente como consecuencia del incremento reciente y continuo de la deforestación en la región del Bosque Chaqueño y del Espinal afín a la actividad agropecuaria. Lo que exigió una mirada alternativa para la integridad ecosistémica (Laclau, 2012).

A esta situación es necesaria sumar la creciente preocupación jurídica-social, que hemos venido desarrollando, que demarca una gran complejidad al momento de pensar en los sistemas de producción. Al imponer un complejo entramado de restricciones generales con la finalidad de integrar y/o pacificar la tensión entre conservación y producción.

En los últimos tiempos distintos agentes públicos y privados, preocupados por el sector pecuario, advirtieron esta situación. Y en paralelo al sistema jurídico estatal, surgió un creciente interés de elaborar mecanismos o instrumentos normativos, como convenios y contratos. En los cuales estos agentes se proponen como objetivos lograr optimizar y aprovechar de forma sustentable la actividad ganadera en forma integrada a los bosques, principalmente el nativo. Sin embargo estos instrumentos, establecen de algún modo un régimen voluntario. En sentido de opción para los agentes -públicos o privados- de someterse o no a las normas técnicas de dichos instrumentos. 
En 2015 la entonces Secretaría de Ambiente y Desarrollo y el Ministerio de Agricultura, Ganadería y Pesca de la Nación, celebraron un convenio marco interinstitucional, de naturaleza técnico-político. El que se concretó en el Plan Nacional de Manejo de Bosque con Ganadería Integrada -MBGI(Ministerio de Agricultura, 2015). Sus principales objetivos son establecer el marco general y los principales lineamientos para que las actividades ganaderas en zonas de $\mathrm{BN}$, con el fin de cumplir los criterios de sustentabilidad ambiental, económica y social, pilares básicos del desarrollo sostenible y presupuesto mínimo según la ley nacional de BN.

El convenido se propone promover el fortalecimiento de las Provincias induciendo la generación de capacidades para la consumación de planes de MBGI para asegurar la provisión de los servicios ecosistémicos y evitar su fragmentación ${ }^{4}$. También se acuerda respetar tres principios básicos de la sustentabilidad en simultaneidad: "1) La capacidad productiva y la productividad del ecosistema deben mantenerse o mejorarse, 2) La integridad del ecosistema y sus servicios deben mantenerse o mejorarse y 3) El bienestar de las comunidades asociadas a su uso debe mantenerse o mejorarse" (Ministerio de Agricultura, 2015).

Otro mecanismo, pero desde el ámbito privado contractual, es el sistema de certificación del sello Carne Carbono Neutro. Concepto-marca desarrollado por Embrapa (Brasil). Un objetivo del sello es certificar la carne bovina, cuyos volúmenes de emisión de GEI fueron contrarrestados durante el proceso de producción por la representación de árboles integrados en sistemas agrosilvopastoril a través de procesos controlados (BuscaAgro, 2015).

El sello tiene un doble sentido económico-ambiental. Por un lado buscar promover el estudio de sistemas de producción ganadera más sostenible, sobre todo con introducción del árbol como factor capaz de neutralizar el metano emitido por el ganado. Y por lo que al mismo tiempo se agrega valor a la carne producida en estos sistemas. Sin embargo tenemos que destacar que esta especie de instrumentos permanece en la esfera del ámbito privado. Siendo solo aplicable aquellos productores que decidan someterse al proceso de certificación y ajuste de su producción ganadera.

Otro objetivo consiste en difundir la importancia estratégica de la sostenibilidad en las cadenas de suministro asociadas (carne, granos, silvicultura), promoviendo el uso de sistemas integrados, optimizando los insumos y factores de producción con efectos sinérgicos positivos. Algunos aspectos importantes en la producción de carne vacuna que se deben considerar, son el bienestar animal, conservación de suelos y agua, y el secuestro de carbono (I. de P. de C. V. Argentina, 2017).

Entre los criterios que deberán cumplir las empresas para la obtención de Marca C-Neutral encontramos la presentación de declaraciones del verificador ante la Dirección de Cambio Climático del Ministerio de Ambiente, con documentación oficial especificada, dicha declaración tiene una vigencia de 3 años y cada año el organismo verificador da seguimiento. De esta manera se propone una tecnificación más compleja de la actividad silvopastoril.

Con estas ideas se revalorizan, desde aspectos económico y ambiental, los beneficios que tiene la inclusión de árboles en sistemas agro-ecológicos, principalmente en el secuestro de carbono, reducción de emisiones de metano y de la presión de los desmontes sobre los bosques (Laclau, 2012). Sin embargo, estos sistema integrativos de bosques y ganadería conforman sistemas complejos de producción. Porque tiene como características su alta complejidad estructural-funcional de manejo y el equilibrio dinámico entre plantas con estructuras y actividad enérgicamente contrastantes como son los árboles y los pastos, y a éstas con el ganado (Carranza, 2016; Laclau, 2012). Complejidad que no permite realizar generalizaciones y dificulta la planificación y monitoreo (INTA, 2015).

\footnotetext{
${ }^{4}$ A la fecha, 10 provincias suscribieron al plan (M. de A. y D. S.-R. Argentina, 2016). Chaco, Santiago del Estero, Salta, Formosa, Chubut, Neuquén, Tierra del Fuego, Santa Cruz y Río Negro (Chubut, 2016).
} 


\section{Conclusión}

Como cierre podemos observar que Corrientes dedica gran parte de su territorio a la producción ganadera la cual afecta al ambiente y contribuye al cambio climático. Pero dicha actividad es económica, social y políticamente esencial en la región.

Sin embargo el derecho ambiental Argentino se consagra en un conjunto de normas jurídicas que complejizan su materialidad limitando y enmarcando la actividad productiva en el paradigma de la sustentabilidad. Esto se ve reflejado en la constitución nacional al incorporar los principios de preservación, reparación y desarrollo sustentable, el deslinde de competencias, entre otros en su art. 41 y los tratados en materia de cambio climático reconocidos por el orden interno, que establecen la responsabilidad de reducir y mitigar los GEI.

En el ámbito de la provincia de Corrientes existen normativas específicas referidas a áreas protegidas y a bosque nativos que establecen límites a la ganadería, con el fin de compatibilizar con la protección al ambiente. Sin embargo estas regulaciones no dan respuesta definitiva que permita calificar a la actividad ganadera como sustentable y no establecen mecanismos ni instrumentos enfocados en mitigar los efectos del cambio climático. Generando una incongruencia entre el sistema jurídico normativo y la realidad.

Específicamente el régimen de bosques nativos establece límites serios a este recurso restringiendo la posibilidad de realizar actividades que alteren el funcionamiento y dinámica del bosque como el sobrepastoreo. Sin embargo se discute si es posible realizar actividad ganadera sustentable. Esto tiene su origen cuando la ley establece en su categoría II de bosque nativo que se podrán realizar actividades compatibles con un manejo sustentable.

En este contexto aparecen diferentes actores preocupados por el sector pecuario que en paralelo al sistema jurídico estatal, elaboran mecanismos o instrumentos normativos alternativos. Como por ejemplo en el sector público el Plan Nacional de Manejo de Bosque con Ganadería Integrada y desde el ámbito privado la certificación de Carne Carbono Neutro.

Sin embargo, por un lado estos sistemas integrativos conforman actividades complejas de producción que dificultan realizar generalizaciones con planificación y monitoreo. Y por otro los instrumentos que lo formalizan establecen de algún modo un régimen solo obligatorio para las partes que se sometan voluntariamente. En sentido de opción para los agentes -públicos o privados- de someterse o no a las normas técnicas de dichos instrumentos.

\section{Bibliografía}

Agritotal. (2017, 9 de marzo). IPCVA y AFOA avanzan hacia la certificación de la carne carbono neutral. Recuperado de http://www.agritotal.com/nota/27303/

Área de Ordenamiento Territorial, Área de Vinculación y Control, \& Ministerio de Ambiente y Desarrollo Sustentable de la Nación. (2015). Informe de estado de implementación 2010-2015: Ordenamiento Territorial de Bosques Nativos y planes alcanzados por el Fondo Nacional para el Enriquecimiento y la Conservación de los Bosques Nativos. Buenos Aires.

Argentina, I. de P. de C. V. (2017). Numero 7 - 03/2017. Carne Argentina, 1-12.

Argentina, M. de A. y D. S.-R. (2016). Informe del estado del Ambiente, 434.

Brundtland, G. H. (1987). Our Common Future. United Nations Commission (Vol. 4). New York. https://doi.org/10.1080/07488008808408783 
Bueno Rubial, M. del P. (2016). El Acuerdo de París: ¿una nueva idea sobre la arquitectura climática internacional? Relaciones Internacionales, 33, 75-95.

BuscaAgro. (2015). El sello "Carne Carbono Neutro": producción de carne sustentable en ambiente tropical. Retrieved from http://www.buscagro.com/detalles/El-sello-_Carne-Carbono-Neutro_produccion-de-carne-sustenta..._73956.html

Cafferatta, N. A. (2012). Tratado, jurisprudencia y doctrina de derecho ambiental. Buenos Aires: La Ley.

Carranza, C. A. (2016). Manejo de bosque nativo con ganadería integrada (MBGI). In XXIV Congreso AAPRESID. Buenos Aires: Resiliar.

Casella, A. P. (1997). Derecho agrario y derecho ambiental. Perspectiva constitucional Argentina. In Cristiani, E. \& Massart Paccini, A. (eds.). Diritto agrario e ambiente. Congresso in omaggio al professor Antonio Carrozza (119-133). Pisa, Italia: Scuola Superiori di Studi Universitari y di Perfezionamento S. Anna.

Chubut, S. de I. P. (2016). Chubut se suma al Plan Nacional de Manejo de Bosque con Ganadería Integrada. Subsecretaria de Información Pública.

Congreso de la Nación Argentina. (1991). Ley 23919. Apruébase una convención relativa a los humedales de importancia internacional especialmente como hábitat de aves acuáticas, firmada en Ramsar. Boletín Oficial, 24-abr(27122), 6.

Congreso de la Nación Argentina. (1994). Ley No 24295. Apruébase la Convención Marco de las Naciones Unidas sobre el Cambio Climático. Boletín Oficial, 11 -ene(27805).

Congreso de la Nación Argentina. (2001). Ley 25438 Apruébase el Protocolo de Kyoto de 1 a Convención Marco de las Naciones Unidas sobre el Cambio Climático, adoptado en Kyoto - Japón. Boletín Oficial, 19-jul(29692).

Congreso de la Nación Argentina. (2002). Ley ${ }^{\circ}$ 25675. Ley general del medio ambiente. Boletín Oficial, 30029(19 de noviembre), 2-4.

Congreso de la Nación Argentina. (2003a). Ley 25.831. Régimen de libre acceso a la información ambiental. Boletín Oficial, 07-ene-200(30312), 1.

Congreso de la Nación Argentina. (2003b). Ley 25688. Régimen de gestión integral de aguas. Boletín Oficial, 30060(3 de enero), 2.

Congreso de la Nación Argentina. (2007). Ley 26.331. Presupuesto mínimos de protección de los bosques nativos. Boletín Oficial, 26-dic(31310), 2.

Congreso de la Nación Argentina. (2010). Ley 26.639. Régimen de Presupuestos Mínimos para la Preservación de los Glaciares y del Ambiente Periglacial. Boletín Oficial, 28-oct(32016), 7.

Congreso de la Nación Argentina. (2017). Ley 27270 . Apruébase el Acuerdo de París. Boletín Oficial, 19-sep(33464).

Convención Constituyente de la Provincia de Corrientes. (2007). Constitución de la Provincia de Corrientes.

Esain, J. J. (2008). Competencias ambientales. Buenos Aires: Abeledo Perrot. 
Gobernador de la Provincia de Corrientes. (1983). Ley 3771. Creación de la reserva natural del Iberá. Boletín Oficial, 15-04.

Gobernador de la Provincia de Corrientes. (2009a). Decreto 1440/09. Establece los objetivos del Parque y Reserva Provincial del Iberá. Boletín Oficial, 26/08/09.

Gobernador de la Provincia de Corrientes. (2009b). Decreto 1966/09. Modifica y complementa el Dto. 1440/09. Boletín Oficial, 24/11/09.

Gordillo, A. A. (n.d.). La Creciente Internacionalización del Derecho, 1-20.

Gordillo, A. A. (2007). Derecho humanos. Buenos Aires: Fund. de Derecho Administrativo.

INTA. (2015). Manejo de bosques con ganadería integrada.

Joaquin, A. N., \& Pérego, J. L. (2001). Sistemas silvopastoriles en el chaco boliviano, 1-7.

Laclau, P. (2012). Consideraciones económicas y ambientales para la toma de decisiones en sistemas silvopastoriles. In Actas del $2^{\circ}$ Congreso Nacional de Sistemas Silvopastoriles (pp. 359-370). Santiago del Estero: Ediciones INTA.

Lucas Garín, A. (2017). Novedades del sistema de protección internacional de cambio climático: el acuerdo de París. Estudios Internacionales (Santiago), 49(186), 137-167. https://doi.org/10.5354/0719-3769.2017.45222

Mahecha, L. (2002). El silvopastoreo: una alternativa de producción que disminuye el impacto ambiental de la ganadería bovina. Revista Colombiana de Ciencias Pecuarias, 15(2), 226-231.

Ministerio de Agricultura, G. y P. (2015). Convenio de articulación institucional entre la secretaría de ambiente y desarrollo sustentable de la jefatura de gabinete de ministros y el ministerio de agricultura, ganadería y pesca. Buenos Aires, Argentina.

Nava Escudero, C. (2016). El acuerdo de París. Predominio del soft law en el régimen climático. Boletín Mexicano de Derecho Comparado, 49(147), 99-135.

PEN, P. E. N. (2009). Decreto 91. Protección Ambiental de los Bosques Nativos. Apruébase la Reglamentación de la ley ${ }^{\circ}$ 26.331. Boletín Oficial, 16-feb( 31595), 1.

Poder Legislativo de Corrientes. (2010). Ley 5974. Bosques nativos. Boletín Oficial, 18/06/10, 9-7.

Preliasco, P., \& Mañarro, F. (2016). La ganadería en el bosque chaqueño, ¿amenaza y parte de la solución? In Di Pangracio, A., Nápoli, A., \& Sangalli, F. (Eds.) Informe Ambiental Anual 2016: Premio Adriana Schiffrin 14va Convocatoria (131-144). CABA: Fundación Ambiente y Recursos Naturales.

Restrepo, P. P., Tobón, D., \& Flórez, J. H. (2008, oct-dic.). Institucionalidad en torno a los mercados de carbono y los mecanismos de flexibilización derivados del Protocolo de Kioto. Revista Facultad de Ingeniería Universidad de Antioquia, 46, 46-57.

Rodríguez, S. L. (2007). Protocolo de Kyoto: debate sobre ambiente y desarrollo en las discuciones sobre cambio climátio. Gestión Y Ambiente, 10(2), 117-129.

Sabsay, D. A. (1997, July). El nuevo artículo 41 de la constitución nacional y la distribución de competencias nación-provincias. Doctrina Judicial, 783-787. Buenos Aires: La Ley.

Sabsay, D. A., \& Di Paola, M. E. (2002). El federalismo y la nueva ley general del ambiente. Anales 
de Legislación Argentina. Boletín Informativo, (32), 47-54.

Santini, E., \& Senetiner, A. (2011). Cambio Climatico Produccion Agropecuaria. Buenos Aires.

Senado y Cámara de Diputados de la Provincia de Corrientes. (2004). Ley 5590. Reglamenta todo lo referente a rozas y quemas en zonas rurales así como también la prevención y lucha contra incendios rurales. Boletín Oficial, 29/09/04.

Senado y Cámara de Diputados de la Provincia de Corrientes. (2010). Ley 5974. Bosques nativos. Boletín Oficial, 18/06/10, 9-7.

Senado y la Cámara de Diputados de la Provincia de Corrientes. (1993). Ley 4736. Régimen que se aplicará a todas las áreas del territorio provincial que sean declaradas Parque Provincial, Monumento Natural o Reserva Natural para su protección y conservación. Boletín Oficial.

Silberman, J. E., Albanesi, A., \& Grasso, D. (2016). Manejo de bosques con ganadería integrada: Impacto en las comunidades microbianas del suelo. Ciencia Del Suelo, 34(2), 211-219.

Steinfeld, H., Gerber, P., Wassenaar, T., Castel, V., Rosales, M., \& De Haan, C. (2009). La larga sombra del ganado. Problemas ambientales y opciones. Roma: FAO. https://doi.org/10.1007/s10666008-9149-3

Vallejos, V., Botana, M., \& Pohl, V. (2009). Transformaciones territoriales y problemas ambientales en la zona de los Esteros del Ibera. In X Jornadasde Investigación (1-13). https://doi.org/ISSN 18500862

Victoria, M. A. (2017, febrero). ¿El acuerdo de París sobre cambio climático recepciona a la agricultura? Revista Iberoamericana de Derecho Agrario, 5, IJ-CCLXIII-498. 